\title{
Electronic Structure of ZnS:Co Semiconductors: X-ray and Optical Spectroscopy Studies
}

\author{
T.P. Surkova ${ }^{a, *}$, V.R. Galakhov ${ }^{a}$ AND M. GodLewski ${ }^{b}$ \\ ${ }^{a}$ Institute of Metal Physics, Russian Academy of Sciences - Ural Division \\ 620219 Yekaterinburg GSP-170, Russia \\ ${ }^{b}$ Institute of Physics, Polish Academy of Sciences, 02-668 Warsaw, Poland \\ Experimental studies of X-ray photoelectron and Co $L_{\alpha} \mathrm{X}$-ray emission \\ spectra of the $\mathrm{ZnS}$ :Co semiconductor were carried out. It was established \\ that $\mathrm{Co}$ ions are in a $\mathrm{Co}^{2+}$ configuration and that the Co $3 d$ impurity states \\ are localized above the top of the valence band by $1.0 \pm 0.2 \mathrm{eV}$.
}

PACS numbers: 71.55.Gs, 78.70.En, 79.60.-i

\section{Introduction}

Interest in the study of the electronic structure of wide-gap II-VI semimagnetic semiconductors ( $\mathrm{ZnS}, \mathrm{ZnSe}, \mathrm{CdTe}$, etc.) increased substantially in recent years, motivated both by the construction of ZnSe-based semiconductor lasers operating in the blue-green region of the spectrum and due to ferromagnetism with high Curie temperature. Doping with $3 d$ elements changes the optical properties of these compounds and gives rise to magnetic effects depending on the energetic position of the $3 d$ ion states and their interaction with the host bands (see, for example, Ref. [1]). Therefore, it is very important to know the positions of the $3 d$ impurity levels.

The positions of the $3 d$ impurity levels relative to the valence band or the conduction band in the II-VI semiconductors have been established by optical absorption measurements [2]. For Mn-doped II-VI semiconductors (ZnS:Mn, ZnSe:Mn, CdTe:Mn) the energy positions of Mn $3 d$ impurity states have been deduced both by means of photoelectron spectroscopy [3-6] and using a combination of X-ray emission Mn $L_{\alpha}$ spectra and the Mn $2 p$ binding energies determined from X-ray photoelectron spectroscopy (XPS) experiments [7, 8]. It was found that Mn $3 d$

* corresponding author; e-mail: surkova@imp.uran.ru 
impurity states are localized at $2-3 \mathrm{eV}$ under the valence-band edge $[5,6,8]$. According to optical data, Co $3 d$ impurity levels should be localized in the forbidden band [2]. Kowalski et al. [9] have carried out measurements of $3 p-3 d$ resonant photoemission spectra for a $\mathrm{Zn}_{0.9} \mathrm{Co}_{0.1} \mathrm{~S}$ semiconductor and found that the Co $3 d$ contribution to the valence band has a wide structure with two maxima: at the edge of the valence band and about $4 \mathrm{eV}$ below the edge. In our work [10] we have presented X-ray photoelectron core-level and valence-band spectra of a $\mathrm{ZnS}$ :Co semiconductor. We have found that cobalt ions are in a $3 d^{7}$ ground-state configuration and that the degree of covalency of a $\mathrm{Co}-\mathrm{S}$ bond in $\mathrm{ZnS}: \mathrm{Co}$ is somewhat lower than for CoS.

In this work we carried out a direct observation of the Co $3 d$ impurity levels in ZnS:Co using X-ray emission spectroscopy (XES). The combination of the $\mathrm{X}$-ray emission and $\mathrm{X}$-ray photoelectron spectroscopies allowed us to determine the position of the Co $3 d$ states relative to the valence-band edges.

\section{Experiment}

Single-crystal samples of $\mathrm{ZnS}$ and $\mathrm{Zn}_{1-x} \mathrm{Co}_{x} \mathrm{~S}(x \approx 0.05)$ were grown by a chemical transport method. X-ray photoelectron spectra were measured using an ESCA spectrometer from Physical Electronics (PHI 5600 ci) with monochromatic $\mathrm{Al} K_{\alpha}$ radiation. The energy resolution was approximately $0.4 \mathrm{eV}$. The pressure in the vacuum chamber during the measurements was below $5 \times 10^{-9} \mathrm{mbar}$. The spectrometer was calibrated using the Au $4 f_{7 / 2}$ signal from an Au-foil $\left[E_{\mathrm{B}}\left(4 f_{7 / 2}\right)=84.0 \mathrm{eV}\right]$. The binding energies were measured relative to the Fermi level of the spectrometer. Single-crystal specimens of $\mathrm{ZnS}$ and $\mathrm{ZnS}$ :Co were investigated after being broken in vacuum. Because the samples are insulators, a charging effect occurred. The spectra were corrected for the charging effect using the carbon $1 s$ line of hydrocarbons $\left[E_{\mathrm{B}}(1 s)=285.0 \mathrm{eV}\right]$ deposited onto the sample surface during the measurements.

An X-ray emission experiment was performed on the same sample at the bulk branch line of beam line I511 at MAX II (MAX-lab National Laboratory, Sweden). The Co $L_{\alpha} \mathrm{X}$-ray emission spectrum was obtained using a high-resolution

TABLE

Co $2 p$ binding energies and energies of maxima Co $L_{\alpha}$ and Co $L_{\ell}$ emission spectra of Co metal and $\mathrm{Zn}_{0.95} \mathrm{Co}_{0.05} \mathrm{~S}(\mathrm{eV})$. Co $3 d$ (XPS) denotes an $\mathrm{X}$-ray photoelectron valence band maximum of pure cobalt. Co $3 d$ (XES) energy positions were obtained by subtraction of the Co $L_{\alpha}$ energies from the Co $2 p_{3 / 2}$ energies.

\begin{tabular}{l|c|c|c|c|c}
\hline \hline Compound & Co $2 p_{3 / 2}$ & Co $L_{\ell}$ & Co $L_{\alpha}$ & Co $3 d$ (XPS) & Co $3 d$ (XES) \\
\hline Co & 778.3 & $677.8^{*}$ & 777.5 & 1.1 & 0.8 \\
$\mathrm{Zn}_{0.95} \mathrm{Co}_{0.05} \mathrm{~S}$ & 779.4 & $677.8^{*}$ & 778.3 & - & 1.1 \\
\hline
\end{tabular}

${ }^{*}$ Taken from Ref. [12]. 
Rowland-mount grazing-incidence grating spectrometer with a two-dimensional detector [11] at an exciting photon energy of $815 \mathrm{eV}$. The measurements were done at room temperature.

For the calibration of Co $L_{\alpha}$ spectra, we have used the Co $L_{\ell}$ line $\left(3 s \rightarrow 2 p_{3 / 2}\right.$ transition) of Co metal (maximum at $677.8 \mathrm{eV}$ [12]). An energy of the $\mathrm{Co} L_{\alpha}$ maximum for pure cobalt was compared with the $3 d-2 p_{3 / 2}$ energy distance measured by means of X-ray photoelectron spectroscopy. The Co $L_{\ell}$ maxima of pure cobalt and $\mathrm{ZnS}$ :Co are equal in energy. The energy position of the Co $L_{\alpha}$ peak of $\mathrm{ZnS}$ :Co is shifted by $0.8 \mathrm{eV}$ toward higher energies as compared with that of Co metal. All the values used for the calibration are given in Table.

\section{Results and discussion}

The X-ray photoelectron Co $2 p$ spectrum of the doped semiconductors $\mathrm{ZnS}$ :Co is shown in Fig. 1. The Co $2 p$ X-ray photoelectron spectrum shows satellites labeled as $B$ and $D$. The complex structure of the Co $2 p$ spectrum is mainly attributed to the charge-transfer processes. The well-screened $2 p^{5} 3 d^{8} \underline{L}$ states correspond to main maxima ( $A$ and $C$ ), and the poorly screened $2 p^{5} 3 d^{7}$ states correspond to the satellites ( $B$ and $D$ ). Here $\underline{L}$ denotes a hole on a ligand. The energy distances between the satellites and the main lines in the Co $2 p$ spectra of $\mathrm{ZnS}$ :Co are approximately the same as for $\mathrm{CoS}$ and $\mathrm{CoO}$ (see [10]). Thus, one can conclude that the cobalt ions in $\mathrm{ZnS}$ : Co have the ground-state configuration $3 d^{7}$. It correlates with the optical and EPR data which have been done previously on these samples [13].

To determine the energy positions of the Co $3 d$ impurity states of $\mathrm{ZnS}$ :Co relative to the valence-band edge, we have used a combination of Co $L_{\alpha} \mathrm{X}$-ray emission and Co $2 p \mathrm{X}$-ray photoelectron spectra. The Co $L_{\alpha}$ spectra reveal $3 d$ partial states in the valence band. The Co $2 p_{3 / 2}$ binding energy (peak $A$ in Fig. 1)

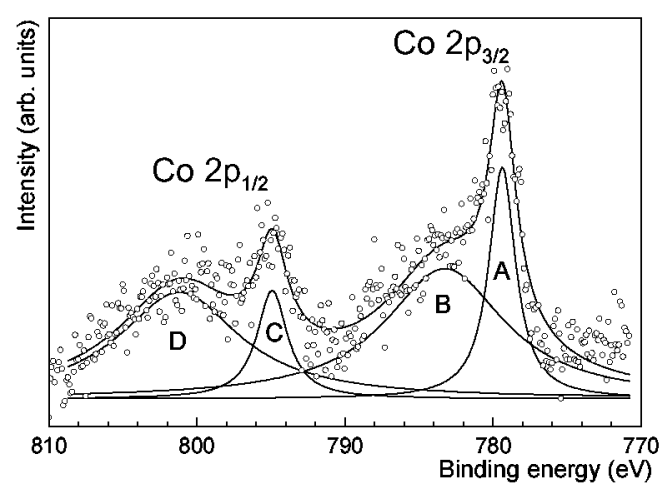

Fig. 1. X-ray photoelectron Co $2 p$ spectrum of ZnS:Co. 


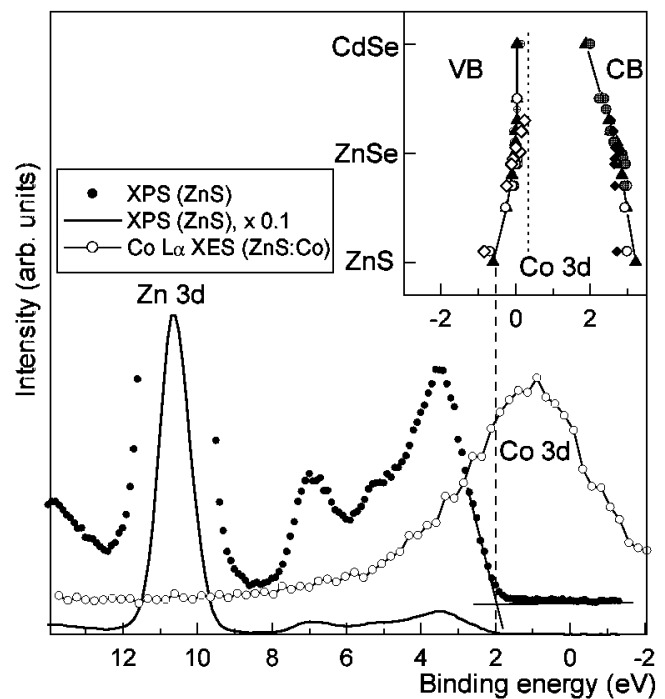

Fig. 2. X-ray photoelectron spectrum of the valence band of $\mathrm{ZnS}$ and Co $L_{\alpha}$ X-ray emission spectrum of $\mathrm{ZnS}$ :Co. The inset presents the position of the Co $3 d$ (optical transition $d^{7} \rightarrow d^{6}$ ) energy level in respect of the valence band and conduction band edges through the whole composition range of the CdSe-ZnSe-ZnS solid solutions obtained using the internal reference rule [17]. The data are taken from the paper of Surkova et al. $[14,15]$ for $T=300 \mathrm{~K}$.

is equal to $779.4 \pm 0.1 \mathrm{eV}$. With this result we can convert the Co $L_{\alpha}$ spectrum of $\mathrm{ZnS}$ :Co into the binding-energy scale, as illustrated in Fig. 2.

The Co $L_{\alpha}$ spectrum of the $\mathrm{ZnS}$ :Co semiconductor is compared with the $\mathrm{X}$-ray photoelectron valence-band spectrum of $\mathrm{ZnS}$. Doping with Co atoms leads to some broadening of the core-level lines as well as of the valence-band spectrum. This occurs due to a distortion of the crystal lattice of the doped crystals and different charge effects for the surface and the volume of the crystals [13]. This effect makes it difficult to determine an exact position of the energy top of the valence band. That is why we have chosen for comparison the spectrum of the undoped crystal. It is necessary to point out that the positions of the $\mathrm{X}$-ray photoelectron peaks do not depend on the Co-impurity concentration [10].

The energy top of the valence band (in the binding-energy scale) is determined by linear extrapolation of the steep edges of the valence-band spectrum to the base line. The top of the valence band, determined in that way, has a binding energy of $2.00 \pm 0.05 \mathrm{eV}$. By using these data we found that the Co $3 d$ impurity states are localized above the top of the valence band by $1.0 \pm 0.2 \mathrm{eV}$.

This result correlates well with the one obtained from optical measurements $[14,15]$ (see also inset in Fig. 2) where the Co $3 d$ impurity level in $\mathrm{ZnS}$ :Co is found to be localized at $0.93 \mathrm{eV}$ above the valence-band edge. 
It is very interesting to compare our results with those obtained by Kowalski et al. [9]. The authors of Ref. [9] have carried out a resonant photoemission study of $\mathrm{Zn}_{0.9} \mathrm{Co}_{0.1} \mathrm{~S}$. A Co $3 d$ contribution to the valence band spectra shows a wide structure with maxima at the edge of the valence band and about $4 \mathrm{eV}$ below the edge. One can see that the Co $3 d$ position found using the complimentary method corresponds well to the first maximum of the resonant photoemission spectrum. It follows from the comparison of the distance between this maximum and the Zn $3 d$ peak. On the other hand, no extend structure is seen in the Co $L_{\alpha} \mathrm{X}$-ray emission spectrum. It should be noted that the $L_{\alpha}$ spectra are not directly related to photoelectron spectra.

The $d$ structure near the edge of the valence band in the resonant photoemission spectrum presented in [9] reveals mainly the $3 d^{7} \underline{L}$ final states, and the area at about $4 \mathrm{eV}$ should be described mainly to the $3 d^{6}$ final states. It follows from the comparison of constant-initial-state (CIS) spectra presented in Ref. [9]. In the CIS spectra, Fano-like resonances are expected from $3 d^{n-1}$ states, whereas antiresonant profiles should be observed from the $3 d^{n} \underline{L}$ states associated with ligand-to-metal charge transfer [16]. The spectrum measured at the binding energy of $1.0 \mathrm{eV}$ has an antiresonant profile, and hence these states should be assigned mainly to the $3 d^{6}$ final states. Similarity, the feature at $-4 \mathrm{eV}$ show both antiresonant and resonant behavior, suggesting that $3 d^{6}$ and $3 d^{7} \underline{L}$ final states overlap in this energy range.

The Co $L_{\alpha}$ spectrum shows only one peak which should be attributed to the transition from the well screened states $2 p^{5} 3 d^{8} \underline{L}$ to $3 d^{7} \underline{L}$. A $3 d^{6}$ contribution to the Co $L_{\alpha}$ spectrum is probably very small and is resulting in a satellite structure. The difference between the photoemission and X-ray emission data can be explained as a result of probing systems with different final states in comparison to the ground state.

\section{Conclusion}

In conclusion, we have determined the position of Co $3 d$ states with respect to the valence band edges by combination of X-ray emission and X-ray photoelectron spectroscopies and found good agreement with the relevant optical data obtained using the concept of the internal reference rule [17]. Co $3 d$ impurity states are localized above the top of the valence band by $1.0 \pm 0.2 \mathrm{eV}$. Our X-ray emission and X-ray photoelectron measurements show that the Co ions are in a $\mathrm{Co}^{2+}$ configuration.

\section{Acknowledgments}

This work was supported by the grants "Cooperation between the Royal Swedish Academy of Sciences and Former Soviet Union States", NATO Collabo- 
rative Linkage Grant (PST.CLG.978044), the Russian Foundation for Basic Research (grant No. 01-02-16991) and RFBR-Ural (grant No. 02-02-96411). Support from the Ultra-Soft X-ray Group of Uppsala University is gratefully acknowledged.

\section{References}

[1] T. Dietl, Semicond. Sci. Technol. 17, 377 (2002).

[2] A. Zunger, in: Solid State Physics, Vol. 39, Eds. H. Ehrenreich, D. Turnbull, Academic Press, New York 1986, p. 275.

[3] D.W. Langer, J.C. Helmer, J. Weichert, J. Lumin. 1,2, 341 (1970).

[4] B.A. Orłowski, K. Kopalko, W. Chal, Solid State Commun. 50, 749 (1984).

[5] L. Ley, M. Taniguchi, J. Ghijsen, R.L. Johnson, A. Fujimori, Phys. Rev. B 35, 2839 (1987).

[6] V.R. Galakhov, T.P. Surkova, V.I. Sokolov, E.Z. Kurmaev, C. Zubrägel, H. Ünlü, S.A. Permogorov, L.N. Tenishev, Solid State Commun. 91, 279 (1994).

[7] W. Zahorowski, E. Gilberg, Solid State Commun. 52, 921 (1984).

[8] V.I. Sokolov, A.N. Mamedov, V.V. Chernyaev, E.Z. Kurmaev, V.R. Galakhov, S.N. Nemnonov, V.P. Kulakov, A.V. Fadeev, Fiz. Tverd. Tela (St. Petersburg) 27, 2118 (1985).

[9] B.J. Kowalski, Z. Gołacki, E. Guziewicz, B.A. Orłowski, J. Mašek, J. Ghijesen, R. Johnson, Acta Phys. Pol. A 86, 831 (1994).

[10] V.R. Galakhov, T.P. Surkova, W. Giriat, S. Bartkowski, M. Neumann, Fiz. Tverd. Tela (St. Petersburg) 39, 1971 (1997) [Phys. Solid State 39, 1762 (1997)].

[11] J. Nordgren, G. Bray, S. Cramm, R. Nyholm, J.-E. Rubensson, N. Wassdahl, Rev. Sci. Instrum. 60, 1690 (1989).

[12] J.A. Bearden, Rev. Mod. Phys. 39, 78 (1967).

[13] K. Świątek, T.P. Surkova, A. Sienkiewicz, A.J. Zakrzewski, M. Godlewski, W. Giriat, Acta Phys. Pol. A 94, 593 (1988).

[14] T.P. Surkova, W. Giriat, S.A. Permogorov, L.N. Tenishev, M. Godlewski, P. Kaszor, A. Zakrzewski, in: Proc. 23 Int. Conf. on Physics of Semiconductors, Eds. M. Scheffler, R. Zimmermann, World Scientific, Singapore 1996, p. 2981.

[15] T.P. Surkova, W. Giriat, M. Godlewski, S. Permogorov, Acta Phys. Pol. A 92, 1009 (1997).

[16] A. Fujimori, F. Minami, S. Sugano, Phys. Rev. B 29, 5225 (1984).

[17] J.M. Langer, C. Delerue, M. Lannoo, H. Heinrich, Phys. Rev. B 38, 7723 (1988). 